\title{
The mechanobiology of kidney podocytes in health and disease
}

\author{
Paul A. Reynolds ${ }^{1,2,3 *}$
}

\author{
Affiliations \\ ${ }^{1}$ School of Medicine, University of St Andrews, St Andrews, UK, KY16 9TF \\ ${ }^{2}$ Biomedical Sciences Research Complex (BSRC), University of St Andrews, St Andrews, UK \\ ${ }^{3}$ Centre of Biophotonics, University of St Andrews, St Andrews, UK \\ *Corresponding Author: Dr Paul Reynolds, par10@st-andrews.ac.uk.
}

\begin{abstract}
(250 words)
Chronic kidney disease (CKD) substantially reduces quality of life and leads to premature death for thousands of people each year. Dialysis and kidney organ transplants remain prevalent therapeutic avenues but carry significant medical, economic and social burden. Podocytes are responsible for blood filtration selectivity in the kidney, where they extend a network of foot processes (FPs) from their cell bodies which surround endothelial cells and interdigitate with those on neighbouring podocytes to form narrow slit diaphragms (SDs). During aging, some podocytes are lost naturally but accelerated podocyte loss is a hallmark of CKD. Insights into the origin of degenerative podocyte loss will help answer important questions about kidney function and lead to substantial health benefits. Here, approaches that uncover insights into podocyte mechanobiology are reviewed, both those that interrogate the biophysical properties of podocytes and how the external physical environment affects podocyte behaviour, and also those that interrogate the biophysical effects that podocytes exert on their surroundings.
\end{abstract}

\section{Introduction}

Conventional thinking in cell biology often assumes that cells communicate mostly via biochemical signaling, but there are many examples to illustrate that cellular forces are ubiquitous in vivo and that these forces directly impact cell function $(1,2)$. Such forces regulate a wide variety of biological processes, e.g. morphogenesis, cell migration, cell proliferation, cell adhesion, differentiation and inflammation. Over a century ago, a number of investigators postulated that forces could regulate cellular processes (1), but these ideas could not be experimentally addressed with the tools available at that time. Importantly, the development of mechanobiology as a field has been closely connected to the advent of enabling technologies. These ideas were only revisited with the establishment of enabling technologies to either measure cellular forces or to observe their effects in vitro $(1,2)$. The biological effects of forces are perhaps most evident in the context of major physiological processes, e.g. breathing, heart function and systemic blood flow, as well as kidney filtration. Indeed, the ability of podocytes to actively attach while being under substantial capillary pressure from the filtration process is key to the function of the healthy kidney (3). 


\section{Podocyte biology}

Podocytes are responsible for blood filtration selectivity in the kidney, where they extend a network of cytoplasmic extrusions from their cell bodies called foot processes (FPs) which surround endothelial cells and interdigitate with those on neighbouring podocytes to form narrow slit diaphragms (SDs) (4). Due to this spatial arrangement, podocytes serve as the final barrier to protein loss in the kidney during filtration of blood by the glomerulus in vivo (Figure 1). This blood filter is under substantial hydrostatic mechanical pressure from blood flow in the glomerular capillaries (5). Two aspects of mechanical force affect podocytes: fluid shear stress (FSS) and stretch. FSS, which is the force per unit area acting parallel to a given surface, occurs on podocyte foot process apical, lateral, and basal surfaces $(6,7)$. Circumferential wall tension in glomerular capillaries due to increased hydrostatic glomerular capillary pressure is countered by the surrounding podocytes and creates stretch in these podocytes (5).

The spatial architecture of the glomerulus adds further complexity to understanding all the mechanical forces that occur within it. The subpodocyte space is a space defined by the glomerular basement membrane (GBM) and the podocyte FPs (with the SD) opposed by the underside of other portions of the podocyte. It is proposed that this space offers significant resistance of flow of primary filtrate. Indeed, it is estimated that up to $60 \%$ of the glomerular filtration apparatus is covered by routes of filtration involving the subpodocyte space (5).

\section{Podocyte pathology}

During aging, some podocytes are lost naturally but accelerated podocyte loss is a hallmark of CKD. Podocyte damage is now recognized as a causal factor in the progression of many different types of kidney disease (8). Podocytes maladapt for survival when they become injured by undergoing a process of foot process effacement (FPE) in which they lose their FP structures, leading to a reduction in filtration barrier function $(3,9)$. FPE is typically associated with the presence of proteinuria. However, not all cases of proteinuria show podocyte FPE, and the structure-function relationship is still not well understood $(3,9)$. There is a spectrum of clinical disease involving the podocyte, each with distinct types of change.

Minimal change disease (MCD) is a glomerular disease resulting in nephrotic syndrome that is usually sensitive to steroid treatment. The pathologic hallmark of disease is the absence of visible alterations by light microscopy and effacement of foot processes by electron microscopy. It accounts for the vast majority of idiopathic nephrotic syndrome cases in children (10). Focal segmental glomerulosclerosis (FSGS) is the most common primary glomerular histologic lesion associated with high-grade proteinuria and with end-stage renal disease (ESRD). Primary FSGS and MCD have many clinical as well as histologic similarities at presentation, making separation into these two categories difficult. Currently, the best method of separation is based on a pathology cut-off, where $>80 \%$ diffuse foot process effacement by electron microscopy demonstrates primary FSGS. However, the correlation with clinical and laboratory parameters, response to therapy and eventual outcome is imprecise (11).

Viruses are capable of inducing a wide spectrum of glomerular disorders. Infectionassociated glomerular nephropathy (GN) commonly includes HCV-associated nephropathy and HIV-associated nephropathy (HIVAN) (12). The classic pathologic hallmark of HCVassociated nephropathy is type 1 membranoproliferative GN (MPGN), which can be resolved if anti-HCV therapy is initiated at an early stage of disease occurrence. HIVAN is characterised by the presence of collapsing FSGS (cFSGS) in the setting of HIV infection (12). Variations in ethnic and APOL1 status affect the rate of HIVAN occurrence. Since 
HIVAN is a direct consequence of viral replication, anti-HIV therapy attenuates the rate of decline of kidney function and causes a return of the normal podocyte phenotype within the glomerular lesions (12).

Membranous nephropathy ( $\mathrm{MN})$ is an autoimmune disease usually associated with a nephrotic syndrome, which is characterized by a thickening of glomerular capillary walls that is visible by optical microscopy. Capillary wall thickening is due to subepithelial deposition or formation of immune complexes (13). Diabetic kidney disease (DKD) develops in approximately $40 \%$ of patients who are diabetic and is the leading cause of CKD worldwide. It results in loss of podocytes with effacement of foot processes, then into segmental and global sclerosis (14).

\section{Methods to exert forces on podocytes}

A number of technological approaches have been used to investigate the response of podocytes to induced extracellular forces. Many of these approaches model the hypertensive kidney, where podocytes need to respond to mechanical forces arising from capillary distention due to increased glomerular capillary hydrostatic pressure.

Ferrimagnetic beads are a method to apply a defined force to cells by applying a magnetic force to move the ferrimagnetic beads in a controlled manner (Figure 2). A study by Eekhoff and colleagues stretched podocytes using an in vitro magnetic bead system (15) where they mechanically stimulated adherent mouse podocytes using the methods of magnetic tweezing and twisting as well as cell stretching. Collagen IV-coated or poly-Llysine-coated magnetic beads were attached to cell receptors to allow the determination of cellular stiffness. The bead-binding strength (defined as the force that is necessary to detach a bead from the cell) showed significant differences between angiotensin II-treated and untreated podocytes. Biochemically, exposing podocytes to cyclic, uniaxial stretch showed an earlier onset of ERK1/2 phosphorylation compared to MEF (control) cells (15). Using similar methods, in response to periodic equibiaxial stretches (10\% in magnitude and $4 \mathrm{sec}$ in duration, spaced $3 \mathrm{~min}$ apart), mouse podocytes harbouring an Actn4 mutation $\left(\right.$ Actn $4^{\mathrm{K} 256 \mathrm{E} / \mathrm{K} 256 \mathrm{E}}$ ) showed an impaired recovery of contraction response compared to wildtype (WT) cells, which corresponded with irreparable disruptions in the actin cytoskeleton (16). Additionally, mutant Actn4 podocytes were at higher risk of detachment after periodic stretch than WT podocytes. Analysis of baseline physical differences between WT and mutant Actn4 podocytes indicated that mutant Actn4 leads to a stiffer actin network as well as a more correlated distribution of actin filament orientation and intracellular stress in the podocyte. These alterations may contribute to the podocyte's vulnerability to stretch and propensity for detachment (16).

A second approach has used mouse podocytes that were cultured on flexible silicone membranes and exposed to mechanical stress by applying vacuum pressure, thereby expanding the cell culture surface and stretching the cells attached upon it (Figure 3). Cultured podocytes exposed to mechanical stretch in vitro using these flexible silicone membranes display mechanosensitive behaviours: the size of podocyte cell bodies and processes become thin and elongated and there is a reorganization of the actin cytoskeleton in both primary rat podocytes and cultured mouse podocytes $(17,18)$. Biochemically, levels of angiotensin II and the AT1R increase, and inhibiting this receptor significantly reduces stretch-induced podocyte apoptosis in both primary and cultured mouse podocytes (19). Fibronectin (Fn1) is highly upregulated by podocytes exposed to mechanical stretch upon flexible silicone membranes and Fn1 influences the expression of specific integrins as well as focal adhesion proteins. Knockdown of Fn1 by siRNAs as well as knockout of Fn1 by CRISPR/Cas9 leads to a high number of lost podocytes upon mechanical stretch, suggesting 
that Fn1 is essential for proper podocyte morphology and function (20). Similar experiments were performed investigating fascin- 1 in stretched podocytes cultured on flexible silicone membranes. Stretched podocytes cultured on flexible silicone membranes for 3 days with 0.5 $\mathrm{Hz}$ and 5\% elongation showed fascin-1 dephosphorylated specifically on Ser-39 under mechanical stretch. This suggests that fascin-1 may play an important role in the adaptation of podocytes to mechanical forces (21). Mechanically stressed podocytes were analysed by cDNA array analysis, which identified the TSG101 gene, as a stretch-induced candidate gene (22). TSG101, which is part of the ESCRT-I complex, is involved in multivesicular body (MVB) formation. TSG101 immunofluorescence was distributed in a vesicular pattern in podocytes, the staining intensity being enhanced by mechanical stress. In DOCA/salt treated rats, a model of glomerular hypertension, glomerular TSG101 mRNA levels were elevated, and an increased number of MVBs were observed by electron microscopy in podocyte processes. These data suggest that mechanical stress upregulates TSG101 in podocytes, and that glomerular hypertension enhances sorting of cell surface proteins and their ligands into the degradative pathway in podocytes (22).

A third approach has investigated the effects of FSS on podocytes (Figure 4). As mentioned above, FSS is the force per unit area acting parallel to a given surface, and it occurs on podocyte foot process apical, lateral, and basal surfaces $(6,7)$. Mouse podocytes respond in a highly sensitive fashion to in vitro shear stress, with a reduction in transversal Factin stress filaments, formation of a cortical actin network and disruption of the cell monolayer $(6,7)$. In an in vivo rat model, biochemically, PGE2 production is increased in podocytes in response to FSS, but not substrate stretch $(7,23)$. Podocytes exposed to FFSS invoke more than one mechanism for mechanotransduction: both Akt-GSK3 $\beta$ - $\beta$-catenin and MAPK/ERK signaling, but not cAMP-PKA signaling (24).

A fourth approach has used gels of tuneable stiffness to alter the mechanical environment of cells (Figure 5). Polyacrylamide gels coated with collagen I have been used to assess podocyte morphology on a range of soft and stiff substrates (25). The authors found that increased substrate stiffness resulted in more mouse podocyte spreading and an increased differentiation phenotype (25). Similarly, Abdallah and colleagues cultured human podocytes on hydrolyzed polyacrylamide (PAAm) hydrogel substrates and observed dense actin cytoskeleton formation and cell spreading with increased substrate stiffness (26). These results were similar to a recent study that investigated whether changes in substrate stiffness affect podocyte morphology and whether optimal substrate stiffness drives podocyte differentiation and biochemical specialization. A tuneable substrate composed of gelatin microbial transglutaminase (gelatin-mTG) was used at a stiffness range $(0.6 \mathrm{kPa}-13 \mathrm{kPa})$ spanning that of healthy and diseased glomeruli. Human podocytes cultured on this substrate showed marked stiffness sensitivity and differing substrate stiffness induced dramatic changes in podocyte phenotype (27). The authors found that a pro-differentiation phenotype was associated with up-regulation of the WT1 transcription factor, a known critical protein involved in a mechanotransduction network, while synaptopodin levels remained unchanged. The authors also tested the Rac/PAK pathway by assessing active phosphorylated (Thr423) p21-activated kinase (PAK) family kinases. They found PAK activity was also modulated by substrate stiffness, showing that Rac-activated PAK is mechano-responsive (27). YAP/TAZ signaling in podocytes has also been assessed in the context of substrate stiffness. Podocytes seeded on both stiff matrices and soft matrices having elastic moduli within the range of those of normal tissues (1.5 to $15 \mathrm{kPa}$ ) displayed a range of phenotypes. Injury upon PAN treatment reduced YAP and TAZ activity in cultured human and mouse podocyte cell lines grown on stiff substrates. Culturing these cells on soft matrix or inhibiting stress fibre formation recapitulated the damage-induced YAP up-regulation observed in vivo, indicating a mechanotransduction-dependent mechanism of YAP activation in podocytes. YAP 
overexpression in cultured podocytes increased the abundance of extracellular matrix-related proteins that contribute to fibrosis (28). A mouse Tg26 model of HIVAN podocyte damage was assessed in the context of an altered matrix environment using polyacrylamide gels coated with type I collagen and collagen matrices that contract when polymerised. The authors found that $\mathrm{Tg} 26$ podocytes cannot recognize rigid matrices and exhibit abnormal responses to their mechanical environment, as suggested by comparison of stress fibre formation and focal adhesion structures with wild-type cells (29). Also, compared to wildtype cells, Tg26 podocytes lack contractile activity, assessed by seeding cells in a threedimensional collagen matrix. These findings suggest that healthy podocytes can take on pathologic characteristics due to the mechanical environment and a pathologically soft environment is sufficient to cause disordered podocyte structure. In milder injury (protamine) models, similar processes occur but over a longer time (29).

A fifth approach involves generating patterned substrates to alter the mechanical environment of cells. A recent article described a substrate consisting of microhemispheres arrayed over a cell culture substrate, which promoted mouse podocyte differentiation in vitro (30). The authors observed increased nephrin expression, structural re-arrangement of F-actin and nephrin within the cell, and increased process formation and interdigitation between cells, compared to flat substrates. Zennaro and colleagues used a nanoporous surface to culture primary rodent and human podocytes (31). The porous surface allowed the formation of mature large and stable punctate focal contacts which appeared after 5 days of incubation and the punctate expression pattern of active vinculin co-localized with actin filaments. These data illustrate the importance of biophysical stimulation in supporting higher-fidelity podocyte cultivation in vitro.

Many of these methodologies use unstressed or unstretched cells as the control comparison. However, even in healthy kidneys, podocytes in vivo are under some mechanical stress. The general limitation of using podocytes in culture is that they are not exposed to a blood stream and thus not exposed to intracapillary pressure and glomerular filtration. More recent advanced in vitro models using iPSCs and/or glomerulus-on-a-chip technology go some way to addressing these issues (32). The limitations of the current technologies described above don't allow for induced extracellular forces on podocytes in vivo. While hypertensive in vivo models show podocyte adaptation and some similarities to the in vitro models, the precise mechanisms of podocyte mechanobiology are yet be defined (33).

\section{Methods to monitor cellular forces exerted by podocytes}

Since podocyte foot processes are too narrow to be resolved by standard light microscopy, where the maximum optical resolution is usually around $200 \mathrm{~nm}$ (34), more advanced techniques are needed to provide the required resolution. Equally, the forces exerted by podocytes and by the foot processes of podocytes in vitro need to be analysed at the required resolution. Several platforms have been developed to measure cellular forces exerted by single cells and cell clusters (35). Atomic force microscopy (AFM) uses a small tip mounted on a flexible cantilever which oscillates at high frequency with the tip in close proximity to the sample surface that either directly or indirectly interacts with the sample (34). AFM is performed to probe the material properties of a cell (36). Welsh and colleagues applied AFM to visualize the cellular surface of cultured podocytes upon application of insulin. They showed that human podocytes rapidly changed their phenotype and retracted their cellular protrusions upon stimulation with insulin (37). Abdallah and colleagues measured the stiffness of human podocyte cultured on PAAm hydrogel, using AFM (26). The stiffest hydrogels produced an increase in human podocyte stiffness. These results are consistent with the observed dense actin cytoskeleton formation and cell spreading with increased substrate stiffness. 
AFM has also been used to investigate the surface stiffness of submicron membrane areas of individual cells (38). In this work, Tandon and colleagues showed that in vitro cultured HIV-infected mouse podocytes were less stiff compared to wild-type cells, indicating severe cytoskeletal changes in the HIV-infected cells. Wyss and colleagues used AFM along with a new technique, capillary micromechanics, to study the mechanical properties of whole isolated rat glomeruli where they measured the elastic properties of the glomeruli (39). The authors showed that glomerular stiffness was reduced upon application of blebbistatin, an agent inhibiting non-muscle myosin IIa/b. Two reagents that inhibit actin polymerization, cytochalasin D and latrunculin B, also reduced glomerular stiffness. Additionally, Col4a3 knockout mice displayed significantly reduced glomerular capillary wall stiffness, compared to wild-type mice, highlighting the essential role of Col4a3 in mechanical stabilization of the GBM to withdraw tensile stress along the glomerular capillary wall. Embry and colleagues used the application of protamine sulphate on cultured podocytes to model mild cytoskeletal changes as seen in MCD, In this setting, they observed a significantly increased plasma membrane indentation, quantified by AFM, in treated podocytes compared to untreated podocytes (29).

Traction force microscopy (TFM) is performed to probe the active contractile prestress of a cell (36), and along with micro-fabricated elastic micro-pillar arrays is the most widely used method for measuring cellular forces in mechanobiology. These approaches are based on tracking the displacement of fluorescent particles embedded in a hydrogel and the bending of elastic pillars, respectively. When pushed to their limits, they achieve a spatial resolution of $2 \mu \mathrm{m}$ (i.e., two distinct forces separated by $<2 \mu \mathrm{m}$ cannot be resolved) and detect cell-induced deformations down to $7 \mathrm{~nm}$ (40) and $25 \mathrm{~nm}$ (41), respectively. Despite their widespread use, both techniques are indirect (i.e. data needs to be analysed offline), they may be photo-toxic to cells and can suffer from swelling, all of which make long-term investigations difficult. Furthermore, data analysis requires zero-cell force reference images and thus removal of cells from the substrate, rendering them unavailable for further analyses, such as immunostaining. Confocal TFM obviates this disruptive step but sacrifices force sensitivity (42). A variant of elastic pillar arrays consists of small diameter $(<100 \mathrm{~nm})$, nanowire arrays fabricated at a high density, increasing the resolution. However, given the diameter and discontinuous nature of the pillars, it is unclear whether cells cultured on nanowire arrays can form adequate-sized FAs (35). Huang and colleagues described a modified method of TFM with optimized regularization and automated Bayesian parameter selection for accurate traction force reconstruction (43). They applied this to the investigation of TFM on primary mouse podocytes and found robust reconstruction of traction forces with this approach.

Kronenberg and colleagues recently described a new technique to measure traction forces: elastic resonator interference stress microscopy (ERISM) (44). ERISM is an interferometric method where cells are grown on an elastic optical micro-cavity consisting of an ultra-soft siloxane-based elastomer (thickness, e.g., $8 \mu \mathrm{m}$ ) sandwiched between two thin, semi-transparent and ultra-flexible gold mirrors (Figure 6). Imaging the reflection of the cavity under illumination with low-intensity monochromatic light of different wavelengths yields fringe patterns from which the cell-induced substrate deformation can be derive with $\mathrm{nm}$ accuracy and $1.6 \mu \mathrm{m}$ lateral resolution. Local stress can be calculated from this data using finite-element-method models. Due to its high precision and simplicity, ERISM could become a viable alternative to study shear and normal forces in 2D cultures. Haley and colleagues have used ERISM to characterize cell forces of differentiated human and mouse podocytes and investigated alterations in podocyte force during stress response in an in vitro model of glomerular injury, thus providing the first experimental demonstration of the importance of podocyte force (3). 


\title{
Conclusions and outlook
}

Development of novel instrumentation and the expansion of the capabilities of current methods will provide enabling technologies to enhance our understanding of podocyte mechanobiology. No current method integrates force measurement with simultaneous fluorescence imaging, and this would be a useful technological development. It would be possible to combine ERISM with super-resolution/single-molecule microscopy for long-term continuous dual measurement and this, along with other enabling technologies, may provide new insights into podocyte biology.

Insights into the origin of degenerative podocyte loss and the role of mechanobiology in this process will help answer important questions about kidney function and lead to substantial health benefits.

\section{Acknowledgements}

P.A.R. acknowledges support from the UK Biotechnology and Biological Sciences Research Council (BB/P027148/1).

\section{Declarations of interest}

The author declares that there are no competing interests associated with the manuscript.

\section{Author contribution statement}

P.A.R. conceived and designed the review, drafted the article, critically revised the article for important intellectual content and performed literature searches.

\begin{abstract}
Abbreviations list
Atomic force microscopy (AFM), chronic kidney disease (CKD), clustered regularly interspaced short palindromic repeats (CRISPR)/CRISPR-associated protein 9 (CRISPR/Cas9), collapsing FSGS (cFSGS), diabetic kidney disease (DKD), elastic resonator interference stress microscopy (ERISM), fibronectin (Fn1), fluid shear stress (FSS), focal segmental glomerulosclerosis (FSGS), foot process (FP), foot process effacement (FPE), gelatin microbial transglutaminase (gelatin-mTG), glomerular basement membrane (GBM), glomerular nephropathy (GN), HIV-associated nephropathy (HIVAN), membranous nephropathy (MN), membranoproliferative GN (MPGN), minimal change disease (MCD), slit diaphragm (SD), multivesicular body (MVB), polyacrylamide (PAAm), traction force microscopy (TFM).
\end{abstract}

\section{References:}

1. Eyckmans J, Boudou T, Yu X, Chen CS. A hitchhiker's guide to mechanobiology. Dev Cell. 2011;21(1):35-47.

2. Mohammed D, Versaevel M, Bruyere C, Alaimo L, Luciano M, Vercruysse E, et al. Innovative Tools for Mechanobiology: Unraveling Outside-In and Inside-Out Mechanotransduction. Front Bioeng Biotechnol. 2019;7:162. 
3. Haley KE, Kronenberg NM, Liehm P, Elshani M, Bell C, Harrison DJ, et al. Podocyte injury elicits loss and recovery of cellular forces. Sci Adv. 2018;4(6):eaap8030.

4. Kriz W. The Inability of Podocytes to Proliferate: Cause, Consequences, and Origin. Anat Rec (Hoboken). 2019.

5. Pichler Sekulic S, Sekulic M. Rheological influence upon the glomerular podocyte and resultant mechanotransduction. Kidney Blood Press Res. 2015;40(2):176-87.

6. Friedrich C, Endlich N, Kriz W, Endlich K. Podocytes are sensitive to fluid shear stress in vitro. Am J Physiol Renal Physiol. 2006;291(4):F856-65.

7. Srivastava T, McCarthy ET, Sharma R, Cudmore PA, Sharma M, Johnson ML, et al. Prostaglandin $\mathrm{E}(2)$ is crucial in the response of podocytes to fluid flow shear stress. J Cell Commun Signal. 2010;4(2):79-90.

8. Assady S, Benzing T, Kretzler M, Skorecki KL. Glomerular podocytes in kidney health and disease. Lancet. 2019;393(10174):856-8.

9. Garg P. A Review of Podocyte Biology. Am J Nephrol. 2018;47 Suppl 1:3-13.

10. Vivarelli M, Massella L, Ruggiero B, Emma F. Minimal Change Disease. Clin J Am Soc Nephrol. 2017;12(2):332-45.

11. Bose B, Cattran D, Toronto Glomerulonephritis R. Glomerular diseases: FSGS. Clin J Am Soc Nephrol. 2014;9(3):626-32.

12. Kupin WL. Viral-Associated GN: Hepatitis C and HIV. Clin J Am Soc Nephrol. 2017;12(8):1337-42.

13. Ponticelli C, Glassock RJ. Glomerular diseases: membranous nephropathy--a modern view. Clin J Am Soc Nephrol. 2014;9(3):609-16.

14. Alicic RZ, Rooney MT, Tuttle KR. Diabetic Kidney Disease: Challenges, Progress, and Possibilities. Clin J Am Soc Nephrol. 2017;12(12):2032-45.

15. Eekhoff A, Bonakdar N, Alonso JL, Hoffmann B, Goldmann WH. Glomerular podocytes: a study of mechanical properties and mechano-chemical signaling. Biochem Biophys Res Commun. 2011;406(2):229-33.

16. Feng D, Notbohm J, Benjamin A, He S, Wang M, Ang LH, et al. Disease-causing mutation in alpha-actinin-4 promotes podocyte detachment through maladaptation to periodic stretch. Proc Natl Acad Sci U S A. 2018;115(7):1517-22.

17. Endlich K, Kriz W, Witzgall R. Update in podocyte biology. Curr Opin Nephrol Hypertens. 2001;10(3):331-40.

18. Martineau LC, McVeigh LI, Jasmin BJ, Kennedy CR. p38 MAP kinase mediates mechanically induced COX-2 and PG EP4 receptor expression in podocytes: implications for the actin cytoskeleton. Am J Physiol Renal Physiol. 2004;286(4):F693-701.

19. Durvasula RV, Petermann AT, Hiromura K, Blonski M, Pippin J, Mundel P, et al. Activation of a local tissue angiotensin system in podocytes by mechanical strain. Kidney Int. 2004;65(1):30-9.

20. Kliewe F, Kaling S, Lotzsch H, Artelt N, Schindler M, Rogge H, et al. Fibronectin is up-regulated in podocytes by mechanical stress. FASEB J. 2019;33(12):14450-60.

21. Kliewe F, Scharf C, Rogge H, Darm K, Lindenmeyer MT, Amann K, et al. Studying the role of fascin-1 in mechanically stressed podocytes. Sci Rep. 2017;7(1):9916.

22. Sunohara M, Kriz W, Kranzlin B, Kretzler M, Gretz N, Endlich K, et al. Upregulation of Tumor Susceptibility Gene 101 (TSG101) by mechanical stress in podocytes. Cell Mol Biol (Noisy-le-grand). 2019;65(1):84-8.

23. Srivastava T, McCarthy ET, Sharma R, Kats A, Carlton CG, Alon US, et al. Fluid flow shear stress upregulates prostanoid receptor EP2 but not EP4 in murine podocytes. Prostaglandins Other Lipid Mediat. 2013;104-105:49-57. 
24. Srivastava T, Dai H, Heruth DP, Alon US, Garola RE, Zhou J, et al. Mechanotransduction signaling in podocytes from fluid flow shear stress. Am J Physiol Renal Physiol. 2018;314(1):F22-F34.

25. Embry AE, Mohammadi H, Niu X, Liu L, Moe B, Miller-Little WA, et al. Biochemical and Cellular Determinants of Renal Glomerular Elasticity. PLoS One. 2016;11(12):e0167924.

26. Abdallah M, Martin M, El Tahchi MR, Balme S, Faour WH, Varga B, et al. Influence of Hydrolyzed Polyacrylamide Hydrogel Stiffness on Podocyte Morphology, Phenotype, and Mechanical Properties. ACS Appl Mater Interfaces. 2019;11(36):32623-32.

27. Hu M, Azeloglu EU, Ron A, Tran-Ba KH, Calizo RC, Tavassoly I, et al. A biomimetic gelatin-based platform elicits a pro-differentiation effect on podocytes through mechanotransduction. Sci Rep. 2017;7:43934.

28. Rinschen MM, Grahammer F, Hoppe AK, Kohli P, Hagmann H, Kretz O, et al. YAPmediated mechanotransduction determines the podocyte's response to damage. Sci Signal. 2017;10(474).

29. Embry AE, Liu Z, Henderson JM, Byfield FJ, Liu L, Yoon J, et al. Similar Biophysical Abnormalities in Glomeruli and Podocytes from Two Distinct Models. J Am Soc Nephrol. 2018;29(5):1501-12.

30. Korolj A, Laschinger C, James C, Hu E, Velikonja C, Smith N, et al. Curvature facilitates podocyte culture in a biomimetic platform. Lab Chip. 2018;18(20):3112-28.

31. Zennaro C, Rastaldi MP, Bakeine GJ, Delfino R, Tonon F, Farra R, et al. A nanoporous surface is essential for glomerular podocyte differentiation in three-dimensional culture. Int J Nanomedicine. 2016;11:4957-73.

32. Veissi S, Smeets B, van den Heuvel LP, Schreuder MF, Jansen J. Nephrotic syndrome in a dish: recent developments in modeling in vitro. Pediatr Nephrol. 2019.

33. Kriz W, Lemley KV. A potential role for mechanical forces in the detachment of podocytes and the progression of CKD. J Am Soc Nephrol. 2015;26(2):258-69.

34. Siegerist F, Endlich K, Endlich N. Novel Microscopic Techniques for Podocyte Research. Front Endocrinol (Lausanne). 2018;9:379.

35. Matellan C, del Río Hernández AE. Where No Hand Has Gone Before: Probing Mechanobiology at the Cellular Level. ACS Biomaterials Science \& Engineering. 2019;5(8):3703-19.

36. Schierbaum N, Rheinlaender J, Schaffer TE. Combined atomic force microscopy (AFM) and traction force microscopy (TFM) reveals a correlation between viscoelastic material properties and contractile prestress of living cells. Soft Matter. 2019;15(8):1721-9.

37. Welsh GI, Hale LJ, Eremina V, Jeansson M, Maezawa Y, Lennon R, et al. Insulin signaling to the glomerular podocyte is critical for normal kidney function. Cell Metab. 2010;12(4):329-40.

38. Tandon R, Levental I, Huang C, Byfield FJ, Ziembicki J, Schelling JR, et al. HIV infection changes glomerular podocyte cytoskeletal composition and results in distinct cellular mechanical properties. Am J Physiol Renal Physiol. 2007;292(2):F701-10.

39. Wyss HM, Henderson JM, Byfield FJ, Bruggeman LA, Ding Y, Huang C, et al. Biophysical properties of normal and diseased renal glomeruli. Am J Physiol Cell Physiol. 2011;300(3):C397-405.

40. Fritzsche J, Albinsson D, Fritzsche M, Antosiewicz TJ, Westerlund F, Langhammer C. Single Particle Nanoplasmonic Sensing in Individual Nanofluidic Channels. Nano Lett. 2016;16(12):7857-64.

41. Wolfenson H, Meacci G, Liu S, Stachowiak MR, Iskratsch T, Ghassemi S, et al. Tropomyosin controls sarcomere-like contractions for rigidity sensing and suppressing growth on soft matrices. Nat Cell Biol. 2016;18(1):33-42. 
42. Bergert M, Erzberger A, Desai RA, Aspalter IM, Oates AC, Charras G, et al. Force transmission during adhesion-independent migration. Nat Cell Biol. 2015;17(4):524-9.

43. Huang Y, Schell C, Huber TB, Simsek AN, Hersch N, Merkel R, et al. Traction force microscopy with optimized regularization and automated Bayesian parameter selection for comparing cells. Sci Rep. 2019;9(1):539.

44. Kronenberg NM, Liehm P, Steude A, Knipper JA, Borger JG, Scarcelli G, et al. Long-term imaging of cellular forces with high precision by elastic resonator interference stress microscopy. Nat Cell Biol. 2017;19(7):864-72.

45. Haraldsson B, Nystrom J, Deen WM. Properties of the glomerular barrier and mechanisms of proteinuria. Physiol Rev. 2008;88(2):451-87.

\section{Figures legends}

Figure 1: Glomerular filtration. Schematic of glomerular filtration showing a podocyte with its foot processes attached to the glomerular basement membrane (GBM). Glomerular filtration rate (GFR) is $125 \mathrm{~mL} / \mathrm{min}$ for kidneys of healthy humans (red arrow) and comprises the net outward pressure of filtration at $10 \mathrm{~mm} \mathrm{Hg}$, consisting of outward glomerular blood hydrostatic pressure at $55 \mathrm{~mm} \mathrm{Hg}$, and inward blood colloid osmotic pressure at $30 \mathrm{~mm} \mathrm{Hg}$ and capsular hydrostatic pressure at $15 \mathrm{~mm} \mathrm{Hg}$. Albumin concentration [Alb], approximate period and separation of foot processes, region of expected nephrin clustering, and force $(\mathrm{F})$ exerted by podocytes on the GBM (blue arrows) are shown. Adapted from (45).

Figure 2: Applying a force using magnetic beads. Ferrimagnetic beads bind to cells via integrins on the cell surface. A magnetic field introduces a torque which causes the bead to rotate and to displace. $\mathrm{M}$ denotes the direction of the bead's magnetic moment. Adapted from (15).

Figure 3: Applying mechanical stress with vacuum pressure. Flexible silicone membranes expand their surface area when vacuum pressure is applied. The attached cells then also stretch.

Figure 4: Applying Fluid Shear Stress. Fluid Shear Stress is applied through a microfluidics device with a regulated liquid flow rate. Cells are then analysed for biochemical and phenotypic changes.

Figure 5: Modulating matrix stiffness. Gel solutions of differing mechanical stiffness are created by modifying the amount of substrate cross-linking.

Figure 6 Using elastic resonator interference stress microscopy (ERISM) to measure traction forces. ERISM uses a layered structure of a micro-cavity with an elastomer layer sandwiched between two ultra-thin gold mirrors. A tuneable light source provides monochromatic illumination and epi-collection to record spatially resolved images of microcavity reflectance that equate to elastomer deformation. The use of ERISM on podocytes is described in (3). 


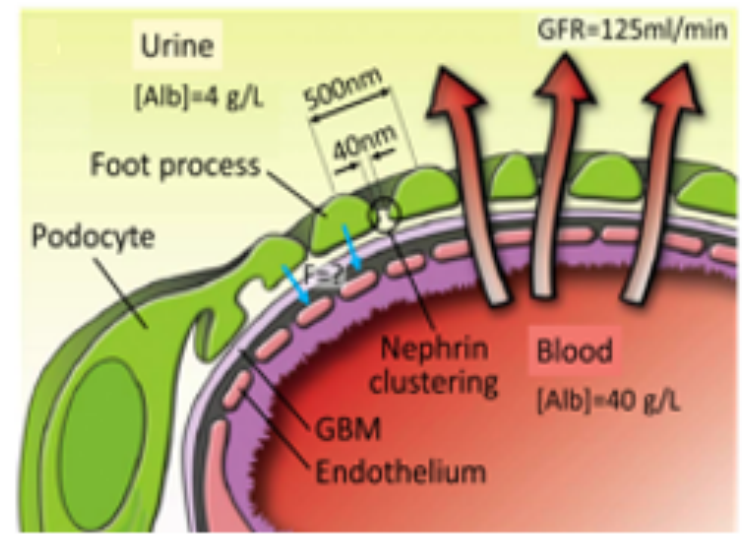




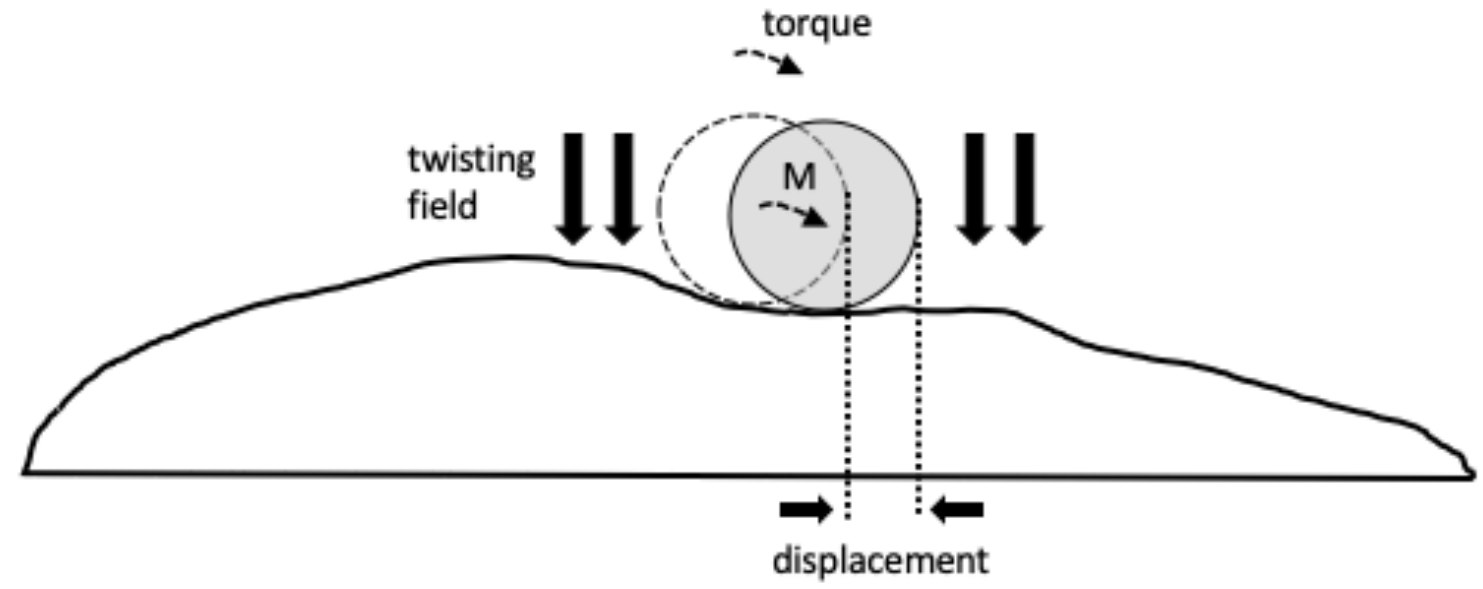




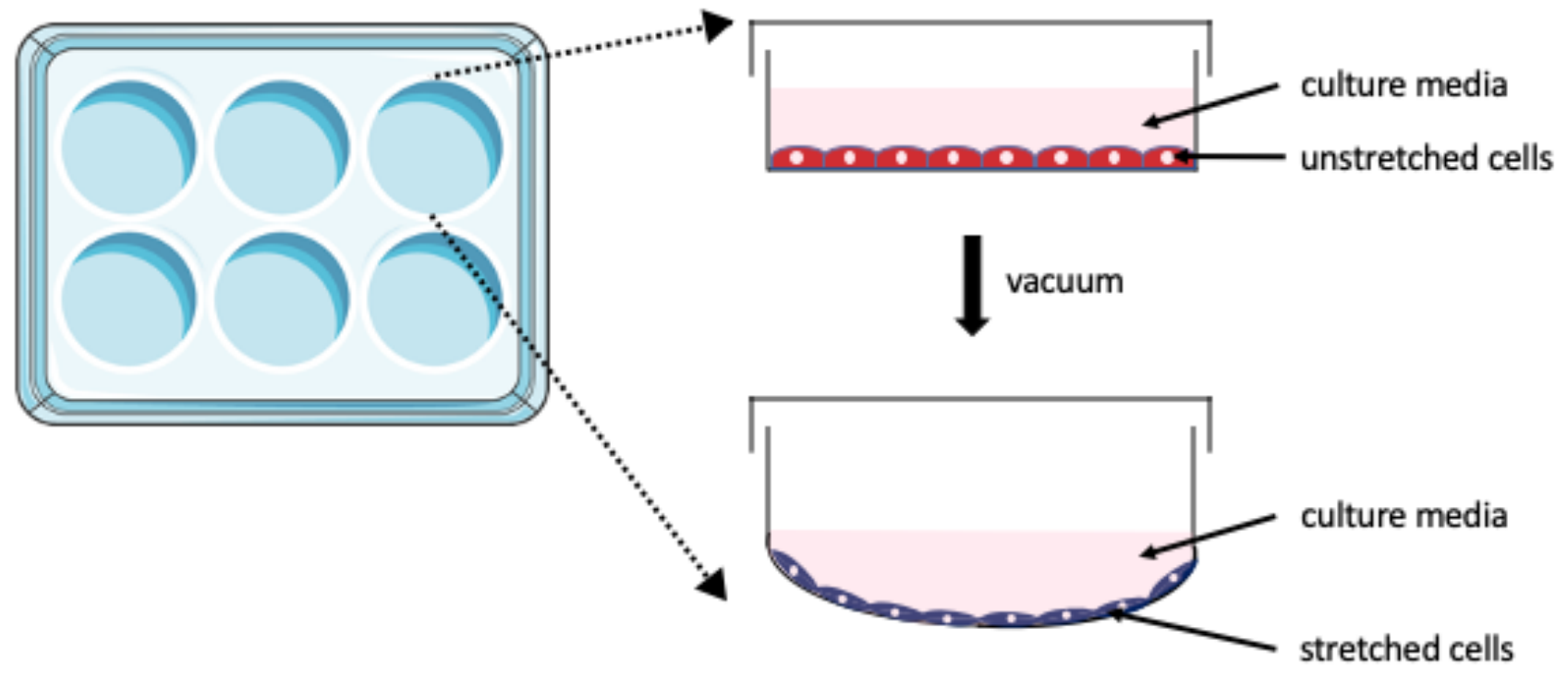




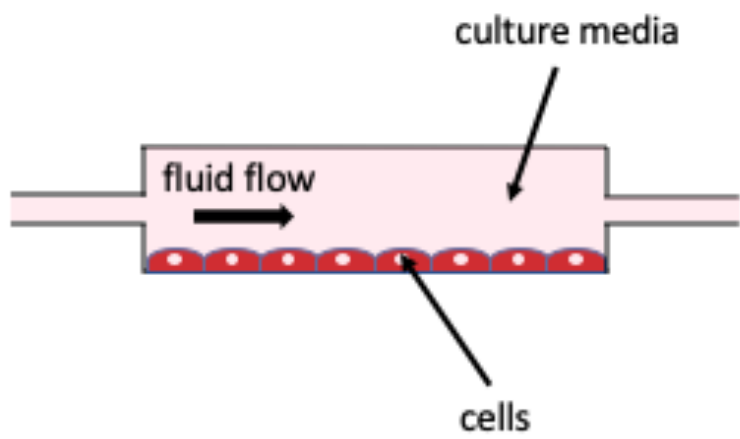




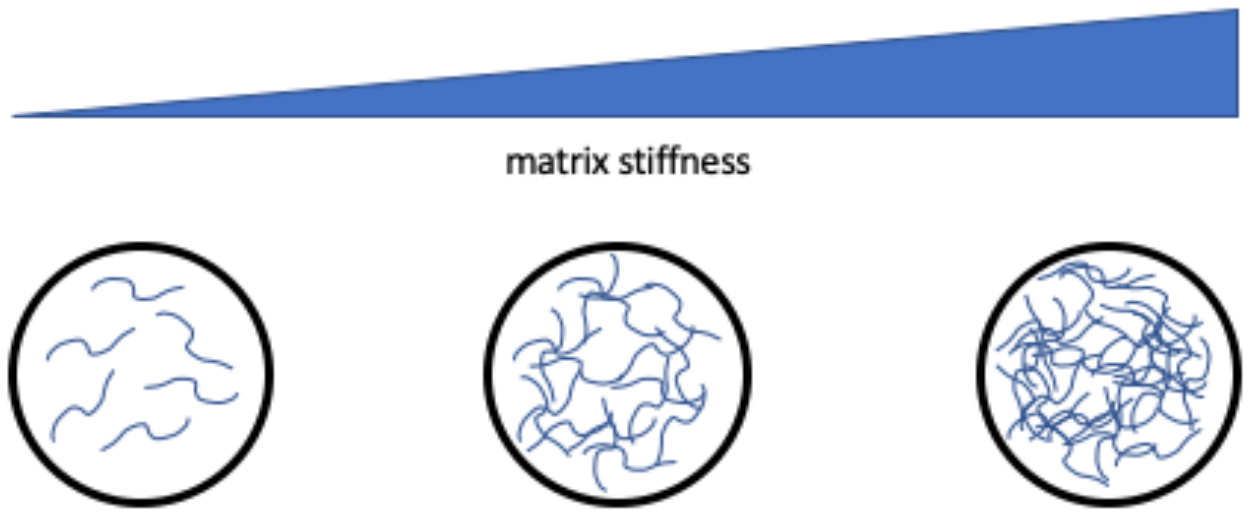




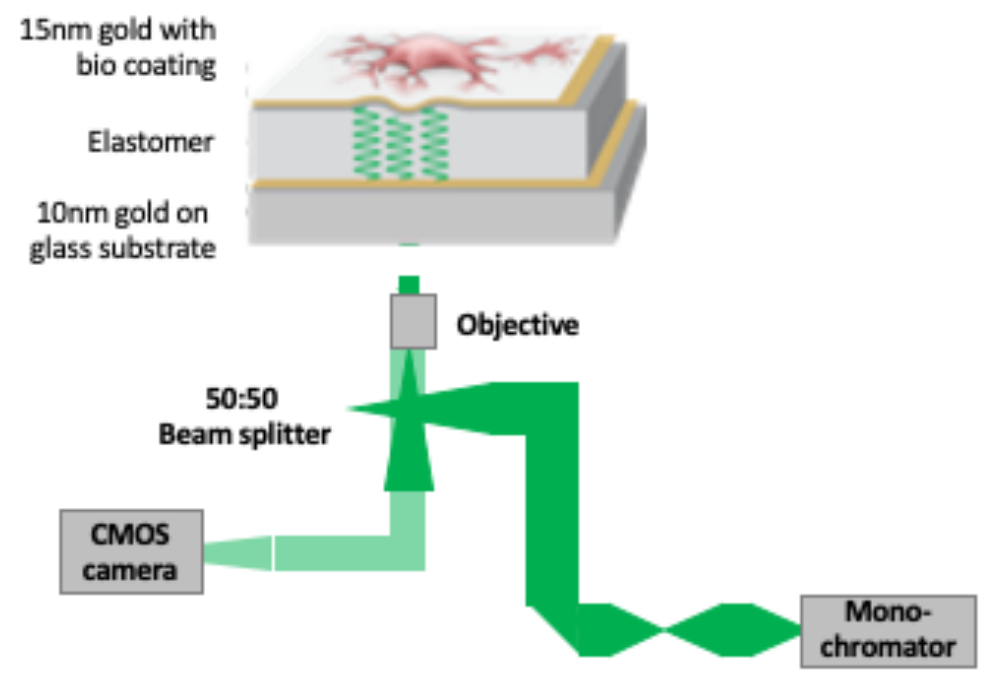

